\title{
Evolução temporal da pandemia do COVID-19 e letalidade em profissionais de saúde no Brasil
}

\author{
Temporal evolution of the COVID-19 pandemic and lethality in healthcare professionals in Brazil
}

Evolución temporal de la pandemia COVID-19 y letalidad en profesionales de la salud en Brasil

\begin{abstract}
RESUMO
Objetivos: Analisar a evolução temporal do COVID-19 e identificar a taxa de letalidade por Síndrome Respiratória Aguda Grave em profissionais de saúde no Brasil. Métodos: Estudo ecológico transversal, com dados secundários no ano de 2020. Foram coletadas informações relacionados aos números de óbitos, casos confirmados e em investigação da COVID-19. Resultados: De janeiro a agosto foram confirmados 1.241 .685 casos de COVID-19 e 4.615 de Síndrome Respiratória entre os profissionais. Destes, 991 foram óbitos, o que resultou em taxa de letalidade de 0,79/1.000. Houve um aumento de $28,7 \%$ no número de casos, entre junho e agosto, enquanto, em agosto, houve uma redução de $33,16 \%$. Na análise de Pearson detectou-se que as correlações significantes foram entre os óbitos por COVID-19 e Síndrome Respiratória. Conclusões: Os resultados além de revelar a real magnitude dos riscos em que se encontra este grupo, apresenta a alerta ao poder público, em todas as suas esferas.
\end{abstract}

DESCRITORES: COVID-19; Saúde Coletiva; Atenção à Saúde do Trabalhador; Síndrome Respiratória Aguda Grave.

\section{ABSTRACT}

Objectives: To analyze the temporal evolution of COVID-19 and to identify the mortality rate due to Severe Acute Respiratory Syndrome in health professionals in Brazil. Methods: Cross-sectional ecological study, using secondary data in the year 2020. Information related to the numbers of deaths, confirmed cases and investigations by COVID-19 were collected. Results: From January to August, 1,241,685 cases of COVID-19 and 4,615 of Respiratory Syndrome were confirmed among professionals. Of these, 991 died, which resulted in a lethality rate of $0.79 / 1,000$. There was an increase of $28.7 \%$ in the number of cases, between June and August, while, in August, there was a reduction of $33.16 \%$. Pearson's analysis showed that significant correlations were between deaths due to COVID-19 and Respiratory Syndrome. Conclusions: The results, in addition to revealing the real magnitude of the risks faced by this group, present the alert to the public authorities, in all its spheres.

DESCRIPTORS: COVID-19; Collective Health; Attention to Occupational Health; Severe Acute Respiratory Syndrome.

\section{RESUMEN}

Objetivos: Analizar la evolución temporal de COVID-19 e identificar la tasa de mortalidad por Síndrome Respiratorio Agudo Severo en profesionales de la salud en Brasil. Métodos: Estudio ecológico transversal, utilizando datos secundarios en el año 2020. Se recopiló información relacionada con el número de muertes, casos confirmados e investigaciones por COVID-19. Resultados: De enero a agosto se confirmaron entre profesionales 1.241 .685 casos de COVID-19 y 4.615 de Síndrome Respiratorio. De estos, 991 murieron, lo que resultó en una tasa de letalidad de 0,79 / 1.000. Hubo un aumento del $28,7 \%$ en el número de casos, entre junio y agosto, mientras que, en agosto, hubo una reducción del 33,16\%. El análisis de Pearson mostró que hubo correlaciones significativas entre las muertes por COVID-19 y el síndrome respiratorio. Conclusiones: Los resultados, además de revelar la magnitud real de los riesgos que enfrenta este colectivo, presentan la alerta a las autoridades públicas, en todos sus ámbitos.

DESCRIPTORES: COVID-19; Salud pública; Atención a la Salud Ocupacional; Síndrome respiratorio agudo severo.

RECEBIDO EM: 19/01/2021 APROVADO EM: 05/02/2021

\section{Cinthia Soares Lisboa}

Mestre em Saúde Coletiva/Universidade Estadual de Feira. Feira de Santana - BA.

ORCID 0000-0002-9370-0465

\section{Leila Magda Rodrigues Almeida}

Mestre em Ciência de Alimentos/Departamento de Análises Bromatológicas, Faculdade de Farmácia, Universidade Federal da Bahia.

ORCID 0000-0002-4496-4309 


\section{Paula Silva Santos}

Pós-graduada em Nutrição Clínica Funcional/Universidade Cruzeiro do Sul e VP Centro de Nutrição Funcional. Pós-graduada em Políticas Públicas/Faculdade Batista Brasileira.

ORCID 0000-0001-6811-9831

\section{Jerusa da Mota Santana}

Doutora em Saúde Pública/Escola de Nutrição/Universidade Federal da Bahia. ORCID 0000-0002-8920-0097

\section{INTRODUÇÃO}

0 Brasil e o mundo enfrentam a mais grave pandemia de uma doença infecciosa que representa um dos maiores desafios sanitários em escala mundial deste século, causada por um novo coronavírus (SARS-CoV-2) ${ }^{1}$. A doença chamada COVID-19 é potencialmente fatal e representa o mais importante problema mundial de saúde pública dos últimos 100 anos, comparado apenas com a gripe espanhola que matou cerca de 25 milhões de pessoas entre 1918 e $1920^{2}$.

No Brasil, a taxa de letalidade em novembro de 2020 (número de mortes pelo total de casos) foi de $2,8 \%$, enquanto que a taxa de mortalidade (quantidade de óbitos por 100 mil habitantes) atingiu 80,5 , e a incidência dos casos de COVID-19 por 100 mil habitantes foi de $2889,1^{3}$.

O novo coronavírus, por sua vez é um dos principais agentes etiológicos que resultam em Síndrome Respiratória Aguda grave (SRAG), além do vírus da influenza A, dengue, vírus sincicial respiratório, adenovírus e hantavírus, e outros agentes (pneumococos, outras bactérias, Legionella sp., leptospirose, etc. $)^{2}$. Com a chegada da COVID-19 e a detecção de transmissão comunitária no país, o protocolo de vigilância de SRAG passou a incluir também o teste para SARS-CoV-2, a partir da $12^{a}$ semana epidemiológica ${ }^{4}$.

São considerados casos de SRAG, devendo ser notificados compulsoriamente, os indivíduos com a combinação dos sintomas de febre alta (acima de $37,8^{\circ} \mathrm{C}$ ) e tosse ou dor de garganta e dificuldade respiratória ou dispneia ou saturação de $\mathrm{O} 2<95 \%$ e necessidade de hospitalização ou óbito tendo apresentado os sintomas referidos, independentemente de hospitalização ${ }^{5}$.

Devido sua elevada transmissibilidade e

\section{O novo coronavírus,} por sua vez é um dos principais agentes etiológicos que resultam em Síndrome

Respiratória Aguda grave (SRAG), além do vírus da influenza $A$, dengue, vírus sincicial respiratório, adenovírus e

hantavírus, $\mathrm{e}$

outros agentes

(pneumococos,

outras bactérias,

Legionella sp.,

leptospirose, etc.) por não existir até o momento fármacos $\mathrm{e}$ vacinas para tratamento e controle da propagação do vírus, a única estratégia eficaz para medida de contenção da COVID-19 é o distanciamento social ${ }^{6}$. No entanto, para os profissionais de saúde que estão no cuidado direto de pacientes diagnosticados com COVID-19 em serviços de atenção primária, nas unidades de pronto-atendimento e nos hospitais, a recomendação de permanecer em casa não se aplica ${ }^{7}$.

Assim, estes profissionais constituem em grupo de risco para a COVID-19 por estarem expostos diretamente aos pacientes infectados, o que propicia maior contato com elevada carga viral ${ }^{7,8}$. Ante o exposto, compreender os dados de mortalidade, principalmente, na população referida, embora as estatísticas de mortalidade sejam criticadas, se faz necessário para monitoramento de saúde pública bem como no planejamento e avaliação da atenção à saúde. Além disso, são dados compreensíveis e aplicáveis vigilância epidemiológica e análise de tendências .

Dados epidemiológicos registram que no Brasil, bem como em outros países, muitos profissionais de saúde foram afastados das atividades laborais por terem se infectado e muitos morreram em consequência da COVID-19 ${ }^{10}$.

Dentre esses profissionais, que estão diretamente ligados a assistência à saúde, destacam-se os técnicos de enfermagem, enfermeiros, médicos, agentes comunitários, agentes de combate a endemias, técnicos de laboratório, fisioterapeutas e farmacêuticos ${ }^{3}$.

Neste contexto, considerando a crescente ocorrência da COVID-19 em profissionais de saúde e a importância desta equipe no enfrentamento da pandemia, faz-se necessário o desenvolvimento deste estudo, que tem como objetivo analisar a 
Gráfico 01. Evolução temporal do número de casos de COVID-19 entre profissionais de saúde no Brasil, Junho a Agosto de 2020.

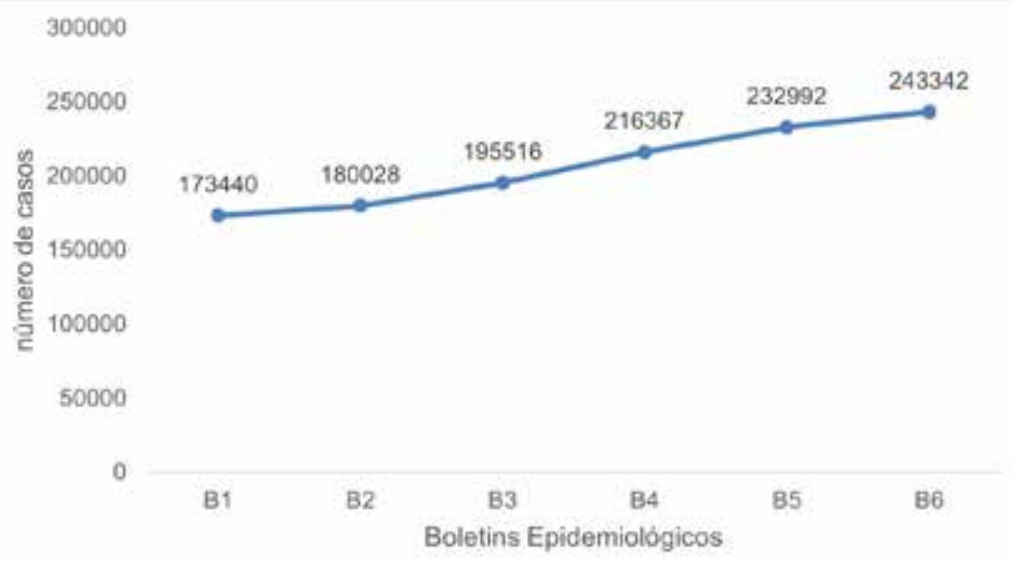

Gráfico 02. Evolução temporal do número de casos de Síndrome Respiratória Aguda Grave (SRAG) em profissionais de saúde no Brasil, Junho a Agosto de 2020.

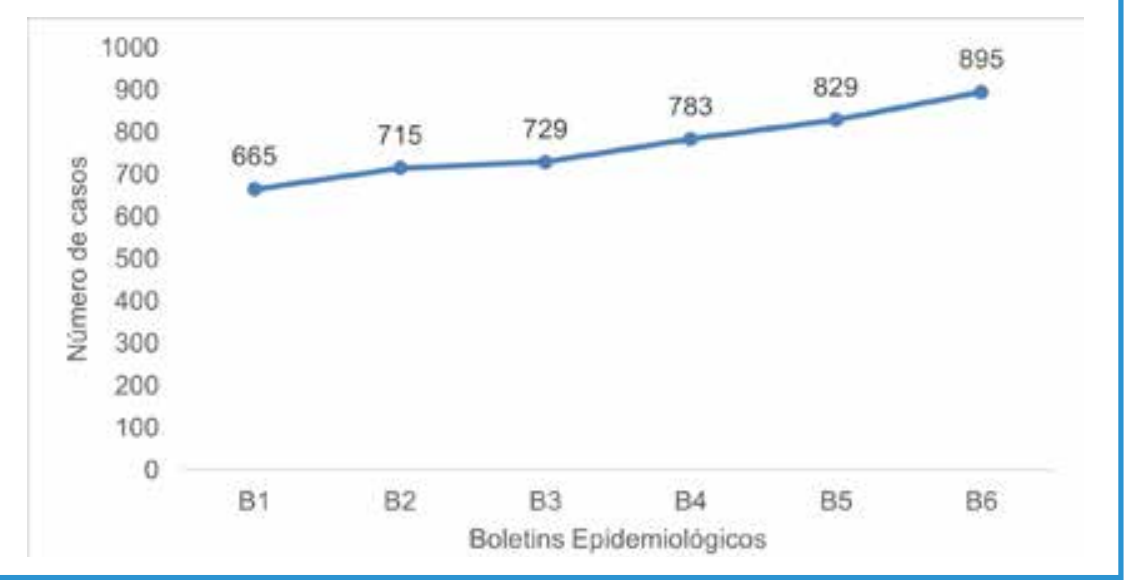

Gráfico 03. Evolução temporal do número de óbitos por COVID-19 em profissionais de saúde no Brasil, Junho a Agosto de 2020.

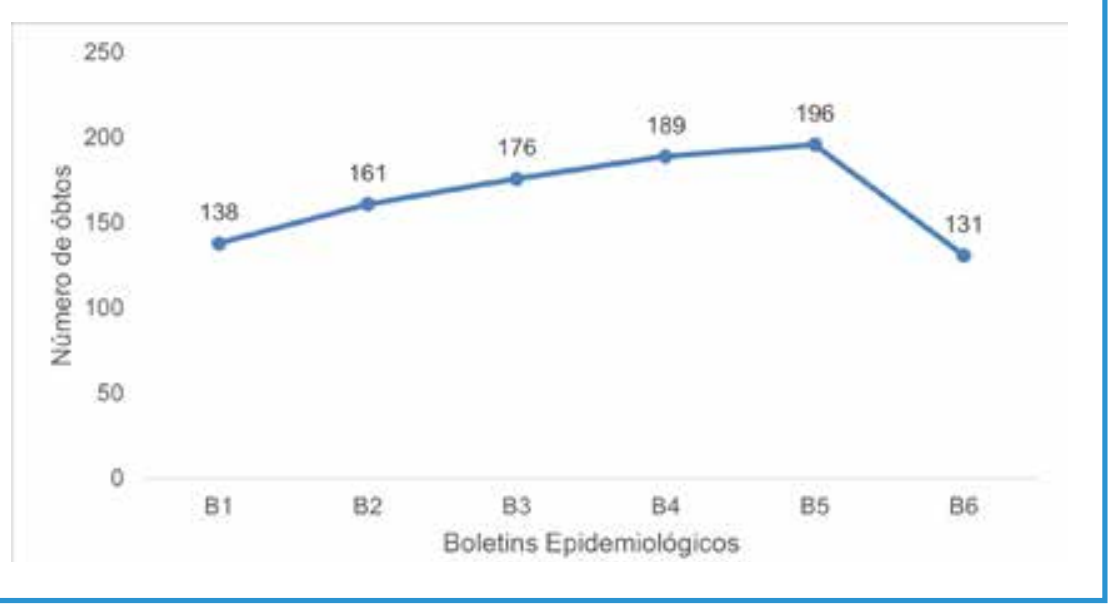

evolução temporal da pandemia da COVID-19 e identificar a taxa de letalidade por SRAG decorrente da doença em profissionais de saúde no Brasil.

\section{MÉTODOS}

Trata-se de um estudo com abordagem metodológica do tipo ecológico, com base de dados secundários provenientes de Sistemas de Informações em Saúde no ano de 2020, tendo o Brasil como unidade de análise.

Foram coletados dados relacionados aos números de óbitos, número de casos confirmados e em investigação da COVID-19 nos profissionais de saúde do Brasil, proveniente do Boletim Epidemiológico COVID-19 - Ministério da Saúde 3 .

$\mathrm{O}$ número de casos confirmados e em investigação em profissionais por SRAG foram coletados no período de janeiro a agosto de 2020. Já os casos de óbitos em profissionais por SRAG foram coletados a partir da $12^{\text {a }}$ semana no boletim epidemiológico (abril a agosto de 2020), período o qual o teste para SARS-CoV-2 foi incluído no protocolo de vigilância em saúde.

Inicialmente foi realizada análise descritiva apresentando as frequências absolutas e relativas das variáveis referentes as profissões de saúde. Utilizou-se o gráfico de linhas para analisar a evolução temporal de número de casos confirmados da COVID-19, número de óbitos da COVID-19 e número de casos da SRAG em profissionais de saúde no Brasil no período estudado. Foi realizado uma Correlação de Pearson da SRAG e óbitos por COVID-19. Considerou-se a relação estatisticamente significante quando valor de $\mathrm{p}<0,05$.

Os dados foram digitados e analisados no programa Statistical Package For The Social Science for Windows (SPSS), versão 17.0.

Este estudo utilizou dados secundários e agregados de domínio público. Portanto, o consentimento livre e esclarecido e a aprovação por Comitê de Ética e Pesquisa estão dispensados, de acordo com a Resolução no 466/2012 da Comissão Nacional de Ética em Pesquisa do Conselho Nacional de Saúde no Brasil. 


\section{RESULTADOS}

Foram identificados no período de janeiro a agosto 1.241 .685 casos confirmados de COVID-19 e 4.615 casos por SRAG entre os profissionais de saúde no Brasil (Gráfico 01 e 02).

Destes 991 evoluiu para óbitos (Gráfico 3) por esta causa, o que resultou em taxa de letalidade de 0,79/1.000 profissionais de saúde. Houve um aumento de 28,7\% no número de casos, entre os meses de junho e agosto, e um aumento de $29,5 \%$ no número de óbitos entre os meses de junho e julho, enquanto, em agosto, houve uma redução de $33,16 \%$.

Os primeiros casos da COVID-19 em profissionais de saúde confirmados no Brasil, segundo o Boletim Epidemiológico COVID-19 - Ministério da Saúde, ocorreram no mês de agosto, sendo registrado também os primeiros óbitos (Tabela 01). No que se refere as categorias de profissionais de saúde, observa-se que $27,68 \%$ são técnicos de enfermagem, $11,98 \%$ enfermeiros e $8,8 \%$ médicos (dados não apresentados na tabela).

$\mathrm{Na}$ análise da correlação de Pearson pode-se detectar que as correlaçôes significantes ( $\mathrm{r}$ ) foram observadas entre os óbitos por COVID-19 e SRAG. A correlação entre ambas as variáveis foi significantemente elevada $(r=0,984 ; \mathrm{p}=0,000)$. As correlações significantes também foram evidenciadas entre os óbitos e número de casos da COVID-19, sendo de $(0,358$; $\mathrm{p}=0,009)$, respectivamente

\section{DISCUSSÃO}

Este estudo sugere que os profissionais de saúde mais acometidos pela COVID-19 são os que estão diretamente ligados a assistência à saúde, destacam-se os técnicos de enfermagem, enfermeiros, médicos, agentes comunitários, fisioterapeutas e farmacêuticos.

Dados da literatura científica revelam que os profissionais da área de saúde têm três vezes mais chances de contrair o vírus do que a população em geral ${ }^{11}$. E quando se restringe ao universo de infectados, ob-

Tabela 1. Primeiros casos e óbitos confirmados de COVID-19 entre profissionais de saúde no Brasil, 2020.

\section{PROFISSIONAIS DE SAÚDE}

Agente Comunitário de Saúde

Agente de Combate a Endemias

Agente de Saúde Pública

Assistente Social

Atendente de enfermagem

Atendente de farmácia

Auxiliar da área social

Auxiliar de farmácia

Auxilair de veterinário

Auxiliar de radiologia

Biólogo

Biomédico

Cirurgião Dentista

Condutor de ambulância

Cuidador de idoso

Cuidador em saúde

Doula

Educador Físico

Enfermeiro

Engenheiro de Alimentos

Engenheiro de segurança do trabalho

Farmacêutico

Físico

Fisioterapeuta

Fonoaudiólogo

Gestores em saúde

Maqueiro

Médico

Médico Veterinário ou Zootecnista

Microscopista ou auxiliar de laboratório da saúde

Naturólogo

Nutricionista

Odontologista

outros

Outro tipo de agente de saúde ou visitador sanitário

Outros profissionais de ensino

Parteira

Pesquisador das ciências biológicas
CASOS

CONFIRMADOS (N)

ÓBITOS (N)

8.030

2.227

1.756

1.714

0

0

1.628

0

0

1.314

227

1.510

2.897

2.287

0

2.212

10

328

25.718

13

220

3.320

672

4.220

537

3.168

1

19.037

517

615

6

1.980

0

0

5.514

899

3

165
5

0

2

1

0

0

0

0

0

0

0

0

0

0

0

0

0

13

0

0

3

0

4

0

0

0

24

1

0

0

0

7

11

0

0

0

0 


\section{artigo}

Lisboa, C.S.; Almeida, L.M.R.; Santos, P.S.; Santana, J.M.;

Evolução temporal da pandemia do COVID-19 e letalidade em profissionais de saúde no Brasil

serva-se que há uma prevalência elevada no grupo em estudo quando comparado a população brasileira. Pesquisa realizada por Kowalski et al. ${ }^{12}$ registra que os profissionais de saúde representam de 3,8\% a 20\% da população infectada, mostrando o grande impacto da doença na força de trabalho.

Estes achados ratificam dados publicados pelo CDC (Centers for Disease Control and Prevention), sobre os casos da COVID-19 em profissionais de saúde nos Estados Unidos, que encontraram uma proporção superior a $75 \%$ dos casos de profissionais da medicina e da enfermagem, dada sua maior exposição nos atendimentos clínicos e emergenciais ${ }^{13}$. No Brasil, até o dia 22 de agosto de 2020, dados da Organização Mundial da Saúde (OMS), indicaram através de um Alerta Epidemiológico que 241 profissionais de saúde vieram a óbito e outros 268.954 mil foram infectados pelo novo coronavírus ${ }^{14}$.

Desse modo, faz-se necessária a reflexão acerca de como o processo e as condiçôes de trabalho podem oferecer riscos aos profissionais de saúde, enquanto potenciais fontes de exposição ao agente etiológico e, indiretamente, a familiares e demais contatos. Maior proximidade e mais tempo de atenção aos infectados, necessários aos seus processos de trabalho, o uso e/ou disponibilidade de Equipamentos de Proteção Individual (EPI) inadequados, e por conseguinte, a exposição às diversas formas de transmissão do patógeno, podem levar ao adoecimento desses profissionais ${ }^{15}$.

Os próprios profissionais de saúde estão sendo desafiados a buscar conhecimento ao mesmo tempo em que atendem aos casos da COVID-19 que sobrecarregam os serviços, realizam o acompanhamento de pacientes com outros problemas de saúde, e se protegem da exposição ao risco de adoecer. Assim, também é avulta a necessidade de conhecimento profissional para enfrentar o contexto de emergência, e salienta-se a importância da circulação de orientações em hábitos de biossegurança para todas as categorias envolvidas no serviço de saúde, a serem adotados nos locais de trabalho ${ }^{16}$. A adoção do comportamento preventivo traz a possibilidade de mitigar o número de in-

\begin{tabular}{lcc} 
Professor & 158 & 0 \\
Profissional de biotecnologia & 320 & 0 \\
Profissional de educação física & & 0 \\
Psicólogo & 1.825 & 1 \\
Químico atuando na área da saúde & 91 & 0 \\
Recepcionista & 7.642 & 0 \\
Socorrista & 485 & 0 \\
Técnico acupuntura, quiropraxia, massoterapia & 47 & 0 \\
Técnico ou auxiliar em radiologia & 0 & 1 \\
Técnico em nutrição & 0 & 0 \\
Técnico de Enfermagem & 1 & 58 \\
Técnico de saneamento & 241 & 0 \\
Técnico de segurança no trabalho & 945 & 0 \\
Técnico eletrotécnica & 197 & 0 \\
Técnico em Alimentos & 697 & 0 \\
Técnico em diagnóstico & 993 & 0 \\
Técnico em farmácia e manipulação farmacêutica & 2.101 & 2 \\
Técnico em imobilização ortopédica & 135 & 0 \\
Técnico em óptica e optometria & 22 & 0 \\
Técnico em prótese ortopédica & 35 & 0 \\
Técnico ou Auxiliar em Enfermagem & 1.965 & 0 \\
Técnico ou auxiliar em odontologia/saúde bucal & 1.988 & 2 \\
Técnicos de laboratório & 935 & 3 \\
Telefonista & 237 & 0 \\
Terapeuta ocupacional & 173.440 & 0 \\
Total & & 0 \\
& 59.635 & \\
\hline
\end{tabular}

fectados e de óbitos.

Ademais, neste estudo foi identificada a redução no número de óbitos no mês de agosto de 2020, que pode estar associada com o maior conhecimento dos profissionais da saúde sobre o comportamento do vírus e manejos de proteção, bem como redução dos números de casos ativos, o que evita a infecção. Resultado este que se assemelhou com as conclusões de Rangel et al. ${ }^{17}$, de que em longo prazo, se pode observar um achatamento da curva epidêmica como consequência das medidas restritivas, a exemplo de distanciamento social.

No que concerne ao panorama da Campanha Nacional de Vacinação contra a COVID-19, atualizado em 12 de janeiro de 2021, por se tratar de uma busca mundial pela tecnologia, produção e aquisição do imunobiológico, a disponibilidade da vacina é inicialmente limitada. Assim, houve a definição de grupos prioritários para a vacinação, com inclusão dos trabalhadores da saúde, por maior risco para agravamento e óbito. Existe a previsão para que todos os trabalhadores da saúde sejam contemplados com a vacinação, entretanto sabe-se que a ampliação da cobertura desse público será gradativa, conforme disponibilidade de vacinas ${ }^{18}$. Neste cenário, ressalta-se que, com base na prudência, ainda não havendo vacinas disponíveis para todos, ou medicamentos comprovadamente eficazes, a única estratégia para desacelerar a pandemia continua sendo o distanciamento.

Avulta-se a ausência de estudos que deem voz a evolução temporal do CO- 
VID-19, por se tratar de uma doença e de uma situação novas, com relatos iniciais, mais precisamente, de dezembro de 2019. Assim, as lacunas de informação e conhecimento se fazem presentes. A indisponibilidade desses dados e de outras variáveis para análise foi uma limitação desta pesquisa.

Sabendo que infecções graves da COVID-19, apresentadas como SRAG, causam um descontrole inflamatório do organismo, o que aponta para um pior prognóstico do indivíduo acometido. Quanto ao processo de infecção, a invasão da célula pelo SARS-CoV-2, causador da COVID-19, ocorre a partir da ligação da proteína $S$ com um receptor enzimático, a enzima conversora de angiotensina 2 (ACE2) na superfície da célula humana. $\mathrm{O}$ vírus então se funde à membrana e libera seu material genético (RNA viral) no interior da célula que, uma vez infectada, começa a fabricar proteínas que auxiliarão na produção de cópias do vírus. Cada célula infectada é capaz de produzir milhões de novas partículas virais, que uma vez liberadas no organismo do hospedeiro, irão infectar outras células sadias ${ }^{19}$.

Todos os indivíduos, apesar da idade, podem ser infectados pelo SARS-CoV-2 e apresentar a SRAG, que é a condição mais grave e a forma da COVID-19 responsável por causar os óbitos e as internações hospitalares. Entretanto, pessoas de meia-idade e idosos são os mais afetados por esta síndrome ${ }^{20}$.

$\mathrm{O}$ presente estudo demonstrou, também, correlações significantes entre COVID-19 e SRAG, o que corrobora com Bastos et al.4, que discutiram que em 2020 a hospitalização por SRAG, desde a detecção do primeiro caso de COVID-19 no Brasil, superou o observado, no mesmo período, em cada um dos 10 anos anteriores, mesmo com o reconhecido atraso de notificação existente. Também foi possível identificar correlação significante entre os óbitos e número de casos da COVID-19 nos profissionais de saúde, o que se dá a sua evolução para formas mais graves de SRAG.

Evento similar, sobre a mortalidade de profissionais de enfermagem, foi discutido por Benito et al. ${ }^{21}$, que pode estar relacionada à várias questões, como por exemplo,

\section{Sabendo que}

infecções graves

da COVID-19,

apresentadas como

SRAG, causam

um descontrole

inflamatório do

organismo, o que

aponta para um

pior prognóstico

do indivíduo

acometido. Quanto

ao processo de

infecção, a invasão

da célula pelo SARS-

CoV-2, causador da

COVID-19, ocorre

a partir da ligação da

proteína $S$ com um

receptor enzimático,

a enzima conversora

de angiotensina 2

(ACE2) na superfície

da célula humana. a complexidade da enfermidade e o desconhecimento de metodologias de combate e controle, disponibilização reduzida de EPIs pelas instituiçóes empregadoras, a elevada carga-horária de trabalho cotidiana, o elevado quantitativo instituído de pacientes a serem atendidos, o reduzido quantitativo de profissionais organizados institucionalmente para $\mathrm{o}$ atendimento, dentre outros.

À luz do exposto, entende-se que é preciso considerar que os números de casos e óbitos ocorridos talvez sejam ainda mais elevados do que os registrados, devido a uma possível subnotificação, bem como pela falta de confirmação dos casos por carência de testagem em massa nos profissionais ${ }^{22}$.

Para assegurar que se tomem as decisões em saúde pública e, particularmente, em saúde do trabalhador, é fundamental conhecer o número de casos e óbitos de COVID-19 entre os profissionais de saúde, o que se configura como ponto forte deste estudo ${ }^{23}$.

Tal conhecimento pode, além de revelar a real magnitude dos riscos em que se encontra esse grupo laboral, trazer o alerta de que o poder público, em todas as suas esferas, necessita de articulação para elaborar, adaptar, implementar e fiscalizar políticas e normativas que busquem garantir a segurança no ambiente de trabalho dos profissionais de saúde, bem como, auxiliar na estimativa de insumos e equipamentos necessários para o atendimento em saúde, de modo a contribuir para o estabelecimento de estratégias para o enfrentamento da pandemia.

\section{CONCLUSÃO}

As evidências compiladas neste estudo demonstram um alto número de profissionais de saúde infectados e que foram a óbito, especialmente no início da pandemia. No entanto, foi possível observar, a partir do mês de agosto, uma tendência decrescente nos casos de óbitos. No que concerne a categoria de profissionais, os técnicos de enfermagem, enfermeiros e médicos foram os mais infectados, sendo a taxa de letalidade maior entre os técnicos de enfermagem. 


\section{artigo}

Lisboa, C.S.; Almeida, L.M.R.; Santos, P.S.; Santana, J.M.;

Evolução temporal da pandemia do COVID-19 e letalidade em profissionais de saúde no Brasil

Desse modo, à guisa de conclusão, é necessário enfatizar a importância de uma maior atenção aos aspectos que envolvam hábitos de biossegurança por esses profissionais, já que essa, não havendo vacina para todos até o momento, continua sen- do a principal estratégia para controle da transmissão, e dos danos causados pelo novo coronavírus. -

\section{REFERÊNCIAS}

1. Johns Hopkins University. Coronavirus COVID-19 Global Cases by Johns Hopkins CSSE [Internet]. Johns Hopkins University; 2020 https://gisanddata.maps. arcgis.com/apps/opsdashboard/index.html\#/ bda7594740fd40299423467b48e9ecf6

2. Rothan HA, Byrareddy SN. The epidemiology and pathogenesis of coronavirus disease (COVID-19) outbreak. J Autoimmun 2020; 109:102433.

3. Brasil. Ministério da Saúde. Painel de casos de doença pelo coronavírus 2019 (COVID-19) no Brasil [Internet]. Brasília: Ministério da Saúde; 2020.

4. Bastos LS, Niquini RP, Lana RM, Villela DAM, Cruz OG, Coelho FC, Codeço CT, Gomes MFC. COVID-19 e hospitalizações por SRAG no Brasil: uma comparação até a 12a semana epidemiológica de 2020. Cad. Saúde Pública 2020; 36(4): e00070120

5. Zhou F, Yu T, Du R, Fan G, Liu Y, Liu Z, et al. Clinical course and risk factors for mortality of adult inpatients with COVID-19 in Wuhan, China: a retrospective cohort study. Lancet 2020; 395:1054-62.

6. West, R., Michie, S., Rubin, G.J. et al. Applying principles of behaviour change to reduce SARS-CoV-2 transmission. Nat Hum Behav 2020; 4: 451-459 https://doi.org/10.1038/s41562020-0887-9.

7. Teixeira, C.F.S, Soares, C.M., Souza, E.A, Lisboa, E.S, Pinto, I.C.M., Andrade, L, Esperidião,MA. A saúde dos profissionais de saúde no enfrentamento da pandemia de Covid-19. Ciência \& Saúde Coletiva 2020; 25: 3465-3474.

8. Medeiros EA. A luta dos pro_ ssionais de saúde no enfrentamento da COVID-19. Acta Paul Enferm. 2020; 33:e-EDT20200003

9. Johansson, L. A. Targeting Non-vious Erros in Death Certificate. Dissertation presented at Uppsala University for the Degree of Doctor of Philosophy, Facult of Medicine, Uppsala, Sweden, 2008.

10. The Lancet. COVID-19: protecting health -care workers. Lancet. 2020; 395(10228):922.

11. Barroso, B. I. L., Souza, M. B. C. A., Bregalda, M. M., Lancman, S., \& Costa, V. B. B. (2020). A saúde do trabalhador em tempos de COVID-19: reflexões sobre saúde, segurança e terapia ocupacional. Cadernos Brasileiros de Terapia Ocupacional. 28(3), 1093-1102. https://doi.org/10.4322/2526-8910.ctoARF2091

12. Kowalski LP, Sanabria A, Ridge JA, Ng WT, de Bree R, Rinaldo A et al. COVID-19 pandemic: effects and evidence-based recommendations for otolaryngology and head and neck surgery practice. Head Neck 2020; 42(6):1259-67.

13. Burrer SL, de Perio MA, Hughes MM, Kuhar DT, Luckhaupt $\mathrm{SE}, \mathrm{McDaniel} \mathrm{CJ}$, et al. Characteristics of health care person- nel with COVID-19 - United States, February 12-April 9, 2020. MMWR Morb Mortal Wkly Rep. 2020; 69(15):477-81. https:// doi.org/10.15585/mmwr.mm6915e6

14. Organización Panamericana de la Salud / Organización Mundial de la Salud. Alerta Epidemiológica: COVID-19 en personal de salud. 31 de agosto de 2020, Washington, D.C. OPS/ OMS. 2020

15. Duarte MMS, Haslett, MIC, Freitas LJA, Gomes NTN, Silva, DCC, Percio J, Wada MY, Fantinato FFST, Almeida WAF, Silva DA, Gava1 C, França GVA, Macário EM, Baêta KF, Malta JMAS, Alves, AJS. Descrição dos casos hospitalizados pela COVID-19 em profissionais de saúde nas primeiras nove semanas da pandemia, Brasil, 2020. Epidemiol. Serv. Saude, Brasília, 29(5): 2020277, 2020.

16. Xiang YT, Jin Y, Wang Y, Zhang Q, Zhang L, Cheung T. Tribute to health workers in China: A group of respectable population during the outbreak of the COVID-19. Int J Biol Sci. 2020;16(10):1739-40.

17. Rangel TF, Diniz Filho JAF, Toscano, CM. Nota técnica 01 Modelagem da expansão espaço-temporal da COVID-19 em Goiás, 2020.

18. Ministério da Saúde [BR]. Informe Técnico. Campanha Nacional de Vacinação contra a Covid-19. Coordenação-Geral do Programa Nacional de Imunizações - Departamento de Imunização e Doenças Transmissiveis - Secretaria de Vigilância em Saúde. Brasília, 18 de janeiro de 2021.

19. Niquini RP, Lana RM, Pacheco AG, Cruz OG, Coelho FC, Carvalho LM, et al. SRAG por COVID-19 no Brasil: descrição e comparação de características demográficas e comorbidades com SRAG por influenza e com a população em geral. Cad. Saúde Pública 2020; 36(7). Disponivel em: https://www.scielosp.org/ article/csp/2020.v36n7/e00149420/pt/\#

20. Araujo LFSC et al. Aspectos clínicos e terapêuticos da infecção da COVID-19 [Internet]. Salvador: Fio Cruz; 2020. [Acesso em: 26 nov. 2020]. Disponível em: https://www.arca.fiocruz. br/handle/icict/40662

21. Benito LAO, Palmeira AML, Karnikowski MGO, Silva ICR. Mortalidade de profissionais de enfermagem pelo Covid-19 no Brasil no primeiro semestre de 2020. Revisa. 2020; 9: 656-68. https://doi.org/10.36239/revisa.v9.nEsp1.p656a668

22. Duprat IP, Melo, GC. Análise de casos e óbitos pela COVID-19 em profissionais de enfermagem no Brasil. Rev Bras Saude Ocup 2020; 45:30.

23. Ribeiro AP, Oliveira GL, Silva LS, Souza ER. Saúde e segurança de profissionais de saúde no atendimento a pacientes no contexto da pandemia de Covid-19: revisão de literatura. Rev Bras Saude Ocup 2020; 45:25. 